\title{
Serum Procollagen Type I N-Terminal Propeptide and Osteocalcin Levels in Korean Children and Adolescents
}

\author{
Ji Seon Choi ${ }^{1}$, Ikchun Park ${ }^{1}$, Soo Jung Lee ${ }^{2}$, Hyo Jin Ju${ }^{3}$, Hyeyoung Lee ${ }^{1}$, and Jayoung Kim ${ }^{1}$ \\ Departments of ${ }^{1}$ Laboratory Medicine, and ${ }^{2}$ Pediatrics, International St. Mary's Hospital, Catholic Kwandong University College of Medicine, \\ Incheon; \\ ${ }^{3}$ Department of Medical Humanities, Catholic Kwandong University College of Medicine, Incheon, Korea.
}

\begin{abstract}
Purpose: Bone markers can be useful for the diagnosis and treatment of skeletal diseases in children and adolescents. Owing to high skeletal growth velocity and rapid bone turnover, children and adolescents have higher bone marker levels than adults. Thus, a valid age- and sex-specific reference should be established for pediatric populations living in similar environments. We aimed to assess the associations of procollagen type I N-terminal propeptide (P1NP) and osteocalcin with age and sex in a group of healthy Korean children and adolescents.

Materials and Methods: The participants (290 boys and 290 girls, age range 0-18 years) were Korean outpatients. Serum P1NP and osteocalcin levels were measured in control materials and patient samples by electrochemiluminescence immunoassay using an automated Cobas e411 analyzer.

Results: Significant age-dependent variations in bone marker levels were observed in both sexes $(p<0.001)$. The highest P1NP levels were observed during the first year of life; thereafter, levels decreased until puberty. There was no postnatal peak for osteocalcin; however, its levels remained higher than the adult reference range throughout childhood. Significant differences were observed between boys and girls $(p<0.05)$, especially between the ages of 12 and 17 years. Cobas e411 results for P1NP showed satisfactory precision and linearity.

Conclusion: We established reference data for P1NP and osteocalcin levels in healthy Korean children and adolescents, as the first and only study of these parameters in pre-adulthood in Korea. Cobas e411-quantified bone markers may be useful for determining bone metabolism indices.
\end{abstract}

Key Words: Children, adolescent, P1NP, osteocalcin, bone marker

\section{INTRODUCTION}

Bone tissue has high metabolic activity owing to its constant re-

Received: July 10, 2019 Revised: September 26, 2019

Accepted: September 30, 2019

Corresponding author: Jayoung Kim, MD, PhD, Department of Laboratory Medicine, International St. Mary's Hospital, Catholic Kwandong University College of Medicine, 25 Simgok-ro 100beon-gil, Seo-gu, Incheon 22711, Korea.

Tel: 82-32-290-3101, Fax: 82-32-280-5520, E-mail: Imkjy7@gmail.com

-The authors have no potential conflicts of interest to disclose.

(c) Copyright: Yonsei University College of Medicine 2019

This is an Open Access article distributed under the terms of the Creative Commons Attribution Non-Commercial License (https://creativecommons.org/licenses/ by-nc/4.0) which permits unrestricted non-commercial use, distribution, and reproduction in any medium, provided the original work is properly cited. modeling (i.e., formation and resorption) throughout one's life. Generally, bone health is assessed using densitometry techniques, including the gold standard of dual-energy X-ray absorptiometry. In children, bone mineral content is commonly used to assess bone mineral deficiency and to estimate fracture risk. However, these are static measures that do not reflect the dynamic nature of bone metabolism. ${ }^{1,2}$ In contrast, biochemical bone markers provide a more dynamic representation of the changes that occur during bone growth and modeling. ${ }^{1,2}$

Biochemical bone markers are classified according to their representative processes, namely formation and resorption. Bone formation markers are expressed during different phases of osteoblast development and reflect osteoblast function and bone formation. These markers include bone alkaline phos- 
phatase, osteocalcin, and procollagen type I N-terminal propeptide (P1NP). The resorption markers are products of bone type 1 collagen degradation: hydroxyproline, pyridinoline, and deoxypyridinoline are degradation products found in urine, while N-terminal and C-terminal crosslinked peptides can be found in both serum and urine. ${ }^{3}$

Exhibiting high skeletal growth velocity and rapid bone turnover, growing children and adolescents show higher levels of bone markers than adults. In these young individuals, bone markers can be useful for investigating skeletal disease and monitoring responses to treatment. However, it may be difficult to clinically interpret marker data because of the influence of various factors on natural growth and maturation. While biochemical markers of bone turnover have been well-studied in post-menopausal women to monitor osteoporosis and are widely used in clinical practice, ${ }^{4}$ they are poorly documented in children and adolescents. ${ }^{5}$ Therefore, age- and sex-specific references should be established for pediatric populations with similar lifestyles living in comparable environments. To that end, it is necessary to assess the relationships between markers of bone formation/ turnover and age/sex among healthy children and adolescents.

Given this background, we investigated the expression levels of the bone formation marker P1NP and the bone turnover marker osteocalcin in an attempt to establish reference values for each as a function of age and sex in healthy children and adolescents in Korea. We also evaluated the assay performance for serum P1NP.

\section{MATERIALS AND METHODS}

\section{Study participants}

The participants $(n=580 ; 290$ boys and 290 girls, age range $0-18$ years) were recruited from Korean outpatients visiting our department for blood tests. They comprised children with noncomplicated acute conditions, mostly allergies, excluding sun allergy and respiratory (bronchitis, pharyngitis, and otitis) inflammation. The exclusion criteria were as follows: medication influencing bone metabolism, dietary restrictions, trauma-related fracture within the previous 2 years, and chronic disease affecting growth and physical development. Pediatricians selected children who were judged to be of normal development, not underweight or obese, according to the criteria for the study. Clinical data were obtained from reviewing patient medical records by laboratory physicians.

\section{Measurement of P1NP and osteocalcin}

Blood samples were collected from an antecubital vein. The serum was separated by centrifugation and was frozen at $-70^{\circ} \mathrm{C}$ for subsequent analysis of P1NP and osteocalcin. All analyses were completed within 1 year of collection. Sample collection, transportation, separation, and storage were performed according to standard laboratory procedures.
P1NP was measured using monoclonal mouse antibodies against P1NP (total P1NP, Roche Diagnostics, Mannheim, Germany) by the electrochemiluminescence immunoassay (ECLIA) on an automated Cobas e411 analyzer (Roche Diagnostics, Mannheim, Germany). Osteocalcin measurement was also performed by the ECLIA method using monoclonal mouse antibodies against N-MID osteocalcin (N-Mid Osteocalcin, Roche Diagnostics, Mannheim, Germany).

\section{Evaluation of analytical performance for P1NP}

We evaluated the analytical performance of a Cobas e411 instrument for serum PINP using control materials and patient samples. Precision was determined using PreciControl Varia Level 1 (approximately $35 \mu \mathrm{g} / \mathrm{L}$ ) and Level 2 (approximately 220 $\mu \mathrm{g} / \mathrm{L}$ ) controls (Roche Diagnostics, Mannheim, Germany) according to the Clinical and Laboratory Standards Institute (CLSI) EP05-A3 guidelines. ${ }^{6}$ The two levels were tested with five replicates per run for 5 consecutive days, with a total of 25 results obtained to determine repeatability and total imprecision. Linearity tests were performed using high- and low-concentration samples within the measuring range provided by the manufacturer according to the CLSI EP06-A guidelines. ${ }^{7}$ High-concentration and low-concentration samples were mixed at ratios of 4:0, 3:1, 2:2, 1:3, and 0:4 to obtain five testable concentrations. Each sample was measured twice, and linearity was evaluated by regression analysis.

\section{Statistical analysis}

The distribution of P1NP was examined for normality using the Kolmogorov-Smirnov Z-test. ${ }^{8}$ In addition to the exclusion criteria, outliers were further checked and eliminated using the Tukey test. ${ }^{9}$ The reference intervals for P1NP and osteocalcin were calculated using a non-parametric percentile method according to the CLSI C28-A3 guidelines, ${ }^{9}$ and results for age groups were presented as upper and lower limits corresponding to the 2.5th and 97.5th percentiles. Two-sample t test or non-parametric Mann-Whitney test was used to compare parameters between boys and girls, while Spearman correlation coefficients expressed the measures of linear association. The chronological age groups were initially used in data partition; the partition criteria were in accordance with the CLSI guidelines. ${ }^{9}$ Age and sex partitions were determined by first visually inspecting the distribution plots, and then statistically verifying with the Harris and Boyd test. ${ }^{10}$ Robust reference intervals of the two bone markers were then calculated. Statistical analyses were performed using SPSS 22.0 (IBM Corp., Armonk, NY, USA) and Excel 2010 (Microsoft Corp., Washington DC, WA, USA). $p<0.05$ was considered statistically significant. For precision studies, both standard deviations and coefficients of variation (CVs) were calculated using LaboStats ver. 1.5.1.0 (Laboratory Medicine Foundation, Seoul, Korea). 


\section{Ethics statement}

The Catholic Kwandong University International St. Mary's Hospital Institutional Review Board approved this study and waived the requirement to obtain informed consent from the study participants (IS16SSSI0023).

\section{RESULTS}

\section{Reference intervals for P1NP and osteocalcin by age and sex}

A total of 580 healthy children and adolescents participated in this study and were divided into groups according to age (0-18 years) and analyzed separately for each year of age. Both sexes were also evaluated separately to observe sex-related differences. The characteristics of the study population in relation to both bone markers are shown in Supplementary Table 1 (only online) (P1NP in subjects 0-18 years) and in Supplementary Table 2 (only online) (osteocalcin in subjects $0-18$ years). The age- and sex-stratified means, standard deviations, and percentile values for PINP and osteocalcin concentrations are shown in Table 1 and Fig. 1.

Significant variations with age were observed for laboratory bone markers in both sexes $(p<0.001)$, as were significant differences between boys and girls $(p<0.05)$. The highest levels of PlNP were observed during the first year of life, after which the levels decreased with some fluctuations until puberty (Supplementary Table 1, only online; Fig. 1). The levels of P1NP in boys tended to increase in puberty, but showed gradual changes and declined with age in girls. However, there was no postnatal osteocalcin peak. An increase in osteocalcin levels was observed in both sexes with age, peaking (with some plateauing) at 11-13 years in boys and at 9-12 years in girls (Fig. 1).

Both bone markers increased with pubertal onset, and significant differences were observed between boys and girls, especially those between the ages of 12 and 17 years (Supplementary Tables 1 and 2, only online; Fig. 1). Serum PINP and osteocalcin were positively correlated $(\mathrm{r}=0.467, p<0.001)$.

\section{Analytical performance for serum P1NP}

Good precision was achieved when evaluating low- and highlevel controls; the repeatability (within-run imprecision) CVs assessed by 25 measurements were $2 \%$ and $0.4 \%$, respectively. The total imprecision (within-laboratory imprecision) CVs were $2.1 \%$ and $0.5 \%$, respectively. This assay was shown satisfactorily to be linear $\left(\mathrm{R}^{2}=0.9999\right)$ within the clinically measurable concentration range from $14.6 \mu \mathrm{g} / \mathrm{L}$ to $1005.5 \mu \mathrm{g} / \mathrm{L}$.

\section{DISCUSSION}

Bone markers are specific bone cell-derived molecules that reflect this tissue's remodeling activity. They can be classified into

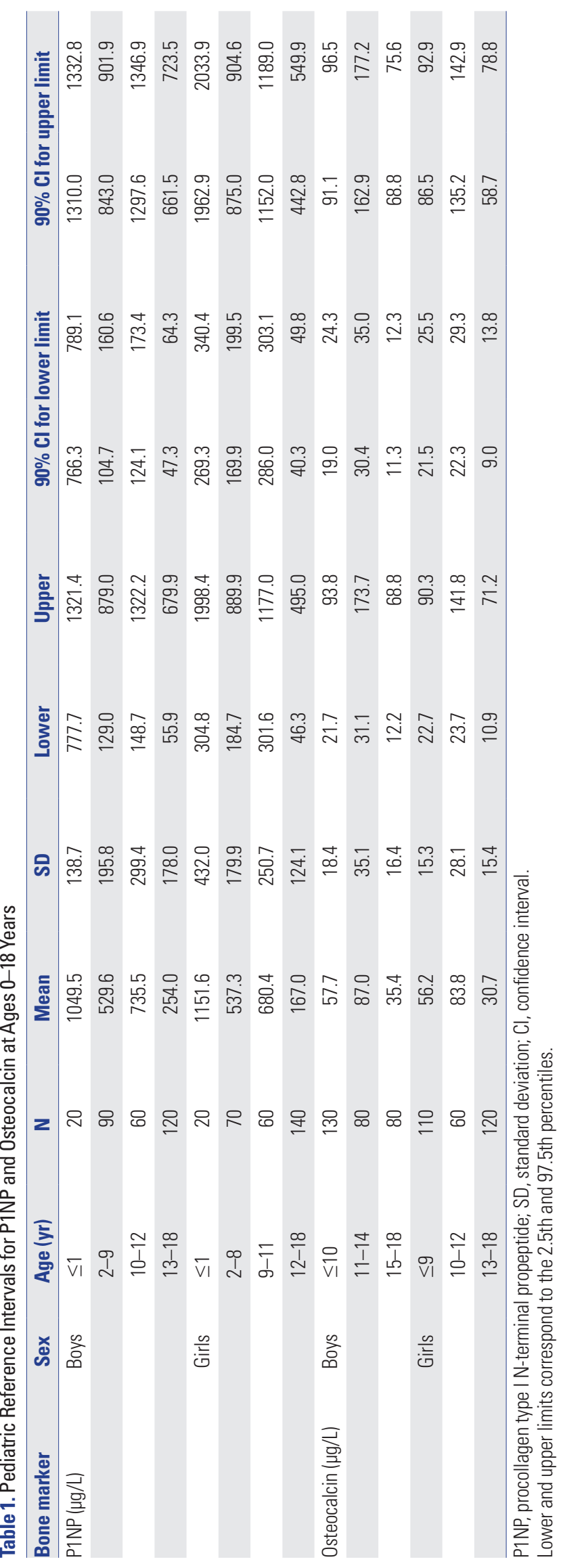



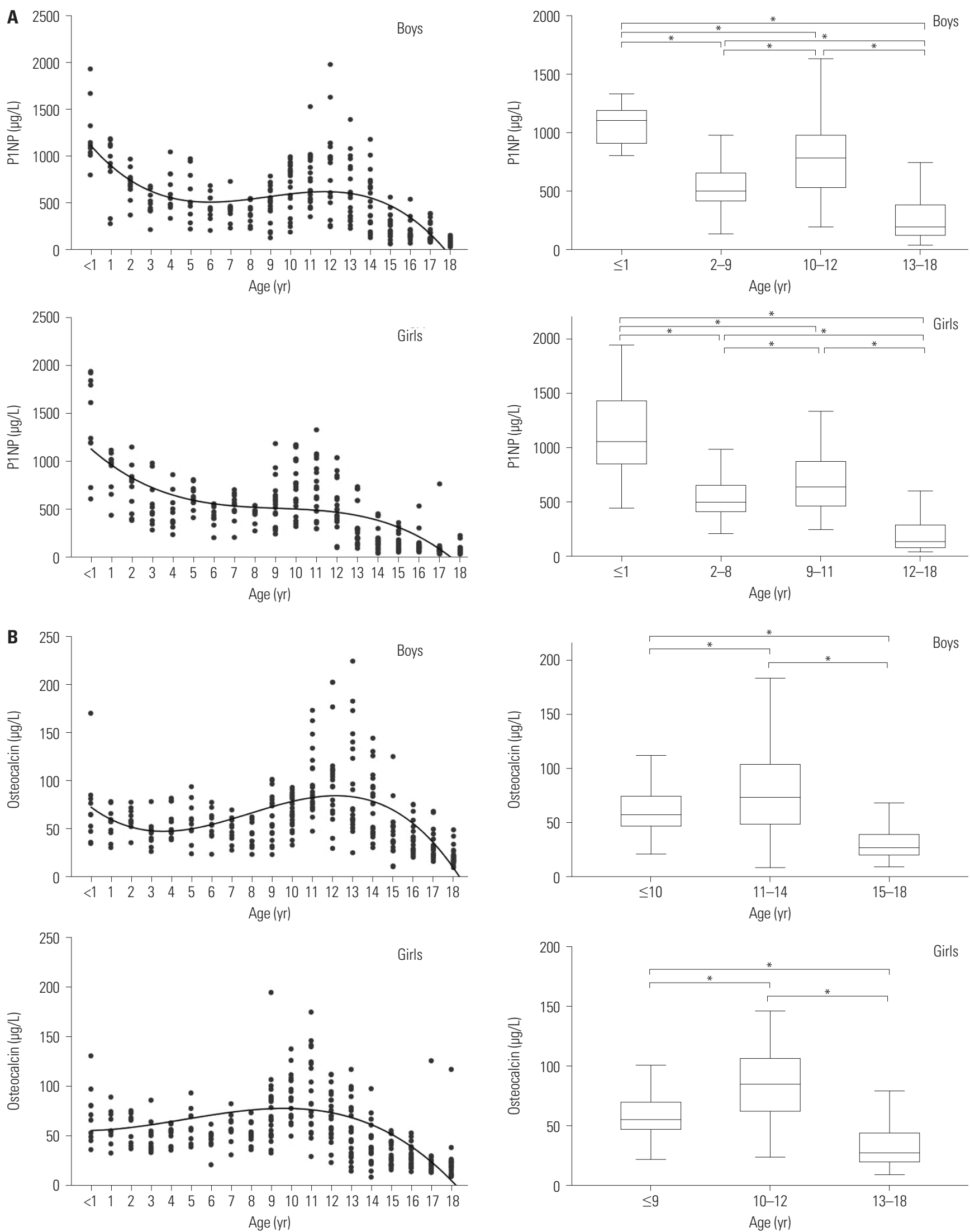

Fig. 1. Left: Distribution of bone marker values according to age. Right: reference intervals of bone markers. The central box represents the values from the lower to upper quartiles (25th to 75th percentile). The middle line represents the median. A line extends from the minimum to the maximum values. (A) Procollagen type I N-terminal propeptide (P1NP) and (B) osteocalcin. * $p<0.05$. 
two categories, bone formation and bone resorption markers. All bone markers are products of bone collagen degradation mediated by osteoclasts during bone resorption and are released into the circulation and excreted through the urine, from whence their levels are usually measured. In children, however, it is difficult to reliably collect a 24-hour urine sample. Concentrations of circulating and urinary markers depend on the amount secreted during bone formation and released from the bone during resorption. Hence, their measured levels do not directly reflect the amount of bone gained during growth. ${ }^{5}$

Type I collagen constitutes approximately $90 \%$ of the organic bone matrix, which is synthesized as procollagen containing $\mathrm{C}$ - and N-terminal extension peptides. They are removed by specific proteases during the conversion of procollagen to collagen and the latter's embedding into the bone matrix. Serum concentrations of P1NP are directly related to the amount of newly formed collagen laid down in the bone. Thus, P1NP is a genuine bone formation marker. P1NP maintains its stability despite repeated freezing and thawing, ${ }^{11}$ and its levels are reproducible and independent of the time of sampling, as no circadian rhythm associations have been documented. ${ }^{12}$ The International Osteoporosis Foundation and International Federation of Clinical Chemistry and Laboratory Medicine Working Group on Bone Marker Standards recommended that serum P1NP be used as the reference marker for bone formation. ${ }^{13}$

Osteocalcin is synthesized by osteoblasts and is the most abundant non-collagenous protein in the bone matrix. After synthesis, a small amount of osteocalcin is released into the circulation. ${ }^{14}$ Circulating osteocalcin has a short half-life and is rapidly cleared by the kidneys. It was previously shown that human serum contains intact osteocalcin molecules, as well as several types of fragments. Serum osteocalcin has been shown to be an important marker of bone turnover, rather than bone formation, as it functions to limit bone formation without impairing bone resorption or mineralization. ${ }^{15,16}$ It is also an excellent indicator of the response to growth hormone therapy. ${ }^{17,18}$ However, serum osteocalcin concentrations have significant circadian-related variations, with its concentrations highest in the morning. ${ }^{18,19}$ Thus, blood samples should strictly be acquired in the morning hours for purposes of osteocalcin measurement. This marker is also relatively unstable; its immunoreactivity reportedly decreases by 50-70\% after 6-24 hours at room temperature and $40-80 \%$ after 2 weeks at $4^{\circ} \mathrm{C} .^{20,21}$ Repeated freezethaw cycles also reduce osteocalcin values. ${ }^{22}$ Moreover, the restricted times during which blood samples could be acquired introduce the possibility of diurnal variations. Seasonal variations, diet, and vitamin $\mathrm{D}_{3}$ supplementation, however, do not influence the expression levels of either P1NP or osteocalcin..$^{23-25}$

The reference intervals we determined for P1NP and osteocalcin varied significantly with age and sex, which was expected given the growth and metabolic activity present during childhood and adolescence. The level of P1NP in boys tended to increase in puberty, but gradually changed and declined with age in girls. During puberty, the ranges of the lower- and upper-limit values of P1NP in boys were higher than those in girls (Korean boys: $55.9-1322.2 \mu \mathrm{g} / \mathrm{L}$ vs. girls, $46.3-1177 \mu \mathrm{g} / \mathrm{L})$. These results are similar to those of a study ${ }^{26}$ comprised of 356 Canadian children aged 6-18 years, in which the ranges of lower- and upperlimit values of P1NP in boys aged $>10$ years were higher than those in girls (Canadian boys, $61-1399 \mathrm{ng} / \mathrm{mL}$ vs. Canadian girls, $38-1346 \mathrm{ng} / \mathrm{mL}$ ). Though comparisons under the age of 10 were not possible due to differences in study-included ages and age stratification, the chronological age changes of bone marker levels were also similar in Korean and Canadian children aged 6-18 years. Meanwhile, osteocalcin had no postnatal peak, although the levels of this marker were higher than the adult reference range throughout childhood.

Compared with the reference intervals with Caucasian children aged 0-18 years reported by Bayer, ${ }^{11}$ the ranges of upper limits (97.5th percentile) of osteocalcin in Korean children were lower in children aged 0-8 years (Caucasian, 85.4-232.5 $\mu \mathrm{g} / \mathrm{L}$. vs. Korean, boys: 67.3-108.8 $\mu \mathrm{g} / \mathrm{L}$; girls: 63.5-113.3 $\mu \mathrm{g} /$ L). For P1NP, the ranges of upper limits in Korean children aged 0-8 years were also lower than Caucasian children (Cau-

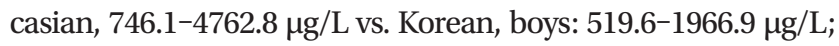
girls: 505.8-2606.7 $\mu \mathrm{g} / \mathrm{L})$. However, the ranges of lower limits (2.5th percentile) of P1NP in both boys and girls aged 9 to 13 years were higher (boys: Caucasian, 45.2-142.5 $\mu \mathrm{g} / \mathrm{L}$ vs. Korean,

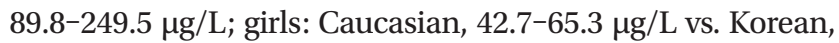
93.7-152.3 $\mu \mathrm{g} / \mathrm{L})$.

One of the challenges of establishing reference intervals in children is the difficulty of recruiting a minimum of 120 participants as advised in the CLSI guidelines. ${ }^{9}$ Since most age groups in our study comprised fewer than 120 individuals, we applied a robust method for calculating the central 95th reference interval percentile as recommended by the guidelines. This involved an iterative process in which observations were downweighted according their distance from the center, and reliable results were generated, despite the small sample size. ${ }^{27}$

Biochemical measurements of bone turnover are essential in the study of the pathophysiology of skeletal metabolism and growth. However, the interpretation of results is difficult because such measurements fluctuate depending on age, pubertal stage, growth velocity, mineral accrual, hormonal regulation, nutritional status, circadian variation, day-to-day variation, and specificity for bone tissue, as well as the sensitivity and specificity of the assays. ${ }^{5,26,28}$ Children of the same sex and chronologic age may yet differ in skeletal age and pubertal stage. As the rates of longitudinal growth and bone remodeling depend on skeletal, chronologic, and pubertal stages, each of these variables must be taken into account when comparing groups of children..$^{29,30}$ Our approach to grouping our participants did not involve classifying them by Tanner stage. The relationship between Tanner stage and chronological age in boys and girls needs to be investigated using a specific tool or self-assessment questionnaire to evaluate the development of pubertal characteristics. How- 
ever, our data also showed that bone markers vary with pubertal growth, with values being higher during early puberty and declining earlier in girls than in boys. Further studies on other covariates that may also influence bone marker values, including physiological factors, such as the body mass index Z-score and parathyroid hormone levels, should also be considered.

Recent advances in research and development have increased the sensitivity, specificity, and availability of assays to detect and measure circulating serum proteins. Therefore, bone markers can be studied for a variety of important purposes, such as basic bone biology research, defining general physiological phenomena in clinical studies or drug trials, and following the medical states of individual patients. Currently, four assays are commercially available for the measurement of circulating PINP, including a radioimmunoassay (RIA) for intact P1NP, an automated chemiluminescence immunoassay for intact P1NP, an enzyme-linked immuno-sorbent assay (ELISA) for total PINP, and an automated ECLIA for total PINP. ${ }^{31}$ The assays specified for intact P1NP preferentially measure the larger PINP antigen in serum, while those labelled for total P1NP are sensitive to both the larger and smaller forms. In this study, we evaluated an automated ECLIA for detecting serum total P1NP with the Cobas e411 instrument and found its analytical performance to be similar to the performances of the Elecsys 2010 automated analyzer (Roche Diagnostics, Mannheim, Germany) ${ }^{32,33}$ and the E170 module immunology analyzer (Roche Diagnostics, Mannheim, Germany). ${ }^{34}$ The repeatability (within-run imprecision) and total imprecision (within-laboratory imprecision) values of Cobas e 411 were $<5 \%$, which are lower than the manufacturer's recommended levels. Cobas e411-quantified bone markers may thus be useful for determining bone metabolism indices.

To compare bone marker data from different studies and laboratories, it is important to establish whether the reference ranges of bone markers are affected by the type of assay used. According to a study comparing bone marker measurements between assays (automated vs. manual), ${ }^{33}$ automated assays appeared to yield lower values than data obtained from manual assays. The type of assay significantly affected the reference intervals obtained for serum cross-linked C-terminal telopeptide of type I collagen and urinary cross-linked $\mathrm{N}$-terminal telopeptides of type I collagen $(p<0.001)$, but not for serum PINP [automated: total P1NP, Elecsys 2010 systems vs. manual: UniQ PINP RIA (Orion Diagnostica, Espoo, Finland)] ( $p=0.280)$. However, RIA has limited routine clinical application because of safety issues. ${ }^{28}$ Of the two assays for total PINP, the manual ELISA is the older one; this method has good precision, but a higher CV than the automated ECLIA. ${ }^{31}$ Fully automated assays allow for rapid and precise determination of bone marker levels.

In summary, to date, there have been no standard Korean reference intervals reported for pediatric bone markers. We determined reference intervals for P1NP and osteocalcin in healthy Korean children living in a similar environment with compara- ble lifestyles. Achieving reliable laboratory bone marker evaluation can help enhance its use in routine clinical practice with the potential for performing such measurements more frequently than bone densitometry. Our new reference intervals may better reflect bone growth and metabolism in children and adolescents. Appropriate interpretation of bone marker values would allow for the early detection of disease progression or evaluation of therapeutic response sooner than can be achieved using bone densitometry.

\section{ACKNOWLEDGEMENTS}

The authors thank Roche Diagnostics Korea for providing the P1NP and osteocalcin assays used in this study.

\section{AUTHOR CONTRIBUTIONS}

Conceptualization: Ji Seon Choi and Jayoung Kim. Data curation: Ikchun Park and Ji Seon Choi. Formal analysis: Ji Seon Choi. Methodology: Ikchun Park and Hyeyoung Lee. Software: Ji Seon Choi and Hyo Jin Ju. Supervision: Jayoung Kim. Validation: Ji Seon Choi, Ikchun Park, and Jayoung Kim. Investigation: Ji Seon Choi and Soo Jung Lee. Writing—original draft: Ji Seon Choi. Writing—review \& editing: Ji Seon Choi and Jayoung Kim.

\section{ORCID iDs}

$\begin{array}{ll}\text { Ji Seon Choi } & \text { https://orcid.org/0000-0002-9179-4517 } \\ \text { Ikchun Park } & \text { https://orcid.org/0000-0001-9314-8773 } \\ \text { Soo Jung Lee } & \text { https://orcid.org/0000-0003-1512-9125 } \\ \text { Hyo Jin Ju } & \text { https://orcid.org/0000-0002-1164-3621 } \\ \text { Hyeyoung Lee } & \text { https://orcid.org/0000-0001-8871-5091 } \\ \text { Jayoung Kim } & \text { https://orcid.org/0000-0003-2977-1813 }\end{array}$

\section{REFERENCES}

1. Jürimäe J. Interpretation and application of bone turnover markers in children and adolescents. Curr Opin Pediatr 2010;22:494-500.

2. Barera G, Beccio S, Proverbio MC, Mora S. Longitudinal changes in bone metabolism and bone mineral content in children with celiac disease during consumption of a gluten-free diet. Am J Clin Nutr 2004;79:148-54.

3. Okabe R, Nakatsuka K, Inaba M, Miki T, Naka H, Masaki H, et al. Clinical evaluation of the Elecsys beta-CrossLaps serum assay, a new assay for degradation products of type I collagen C-telopeptides. Clin Chem 2001;47:1410-4.

4. Khadka B, Tiwari ML, Gautam R, Timalsina B, Pathak NP, Kharel $\mathrm{K}$, et al. Correlates of biochemical markers of bone turnover among post-menopausal women. JNMA J Nepal Med Assoc 2018;56:754-8.

5. Szulc P, Seeman E, Delmas PD. Biochemical measurements of bone turnover in children and adolescents. Osteoporos Int 2000;11:281-94.

6. Clinical and Laboratory Standards Institute. Evaluation of precision of quantitative measurement procedures; approved guideline. 3rd ed. EP05-A3. Wayne (PA): Clinical and Laboratory Standards Institute; 2014.

7. Clinical and Laboratory Standards Institute. Evaluation of the linearity of quantitative measurement procedures: a statistical approach; approved guideline. EP06-A. Wayne (PA): Clinical and 
Laboratory Standards Institute; 2003.

8. Reed AH, Henry RJ, Mason WB. Influence of statistical method used on the resulting estimate of normal range. Clin Chem 1971; 17:275-84.

9. Clinical and Laboratory Standards Institute. Defining, establishing, and verifying reference intervals in the clinical laboratory; approved guideline. 3rd ed. C28-A3. Wayne (PA): Clinical and Laboratory Standards Institute; 2008.

10. Harris EK, Boyd JC. On dividing reference data into subgroups to produce separate reference ranges. Clin Chem 1990;36:265-70.

11. Bayer M. Reference values of osteocalcin and procollagen type I $\mathrm{N}$-propeptide plasma levels in a healthy Central European population aged 0-18 years. Osteoporos Int 2014;25:729-36.

12. Wolthers OD, Heuck C, Heickendorff L. Diurnal variations in serum and urine markers of type I and type III collagen turnover in children. Clin Chem 2001;47:1721-2.

13. Vasikaran S, Cooper C, Eastell R, Griesmacher A, Morris HA, Trenti T, et al. International Osteoporosis Foundation and International Federation of Clinical Chemistry and Laboratory Medicine position on bone marker standards in osteoporosis. Clin Chem Lab Med 2011;49:1271-4.

14. Calvo MS, Eyre DR, Gundberg CM. Molecular basis and clinical application of biological markers of bone turnover. Endocr Rev 1996;17:333-68.

15. Akesson K. Biochemical markers of bone turnover. A review. Acta Orthop Scand 1995;66:376-86.

16. Ducy P, Desbois C, Boyce B, Pinero G, Story B, Dunstan C, et al. Increased bone formation in osteocalcin-deficient mice. Nature 1996;382:448-52.

17. Tobiume H, Kanzaki S, Hida S, Ono T, Moriwake T, Yamauchi S, et al. Serum bone alkaline phosphatase isoenzyme levels in normal children and children with growth hormone (GH) deficiency: a potential marker for bone formation and response to GH therapy. J Clin Endocrinol Metab 1997;82:2056-61.

18. Nielsen HK, Jørgensen JO, Brixen K, Møller N, Charles P, Christensen JS. 24-h profile of serum osteocalcin in growth hormone (GH) deficient patients with and without GH treatment. Growth Regul 1991;1:153-9.

19. Heuck C, Skjaerbaek C, Wolthers OD. Diurnal rhythm of serum osteocalcin in normal children. Acta Paediatr 1998;87:930-2.

20. Banfi G, Daverio R. In vitro stability of osteocalcin. Clin Chem 1994;40:833-4.

21. Garnero P, Grimaux M, Seguin P, Delmas PD. Characterization of immunoreactive forms of human osteocalcin generated in vivo and in vitro. J Bone Miner Res 1994;9:255-64.

22. Power MJ, O'Dwyer B, Breen E, Fottrell PF. Osteocalcin concentrations in plasma prepared with different anticoagulants. Clin Chem 1991;37:281-4.

23. Blumsohn A, Naylor KE, Timm W, Eagleton AC, Hannon RA, Eastell R. Absence of marked seasonal change in bone turnover: a longitudinal and multicenter cross-sectional study. J Bone Miner Res 2003;18:1274-81.

24. Schou AJ, Heuck C, Wolthers OD. Vitamin D supplementation to healthy children does not affect serum osteocalcin or markers of type I collagen turnover. Acta Paediatr 2003;92:797-801.

25. Clowes JA, Hannon RA, Yap TS, Hoyle NR, Blumsohn A, Eastell R. Effect of feeding on bone turnover markers and its impact on biological variability of measurements. Bone 2002;30:886-90.

26. Huang Y, Eapen E, Steele S, Grey V. Establishment of reference intervals for bone markers in children and adolescents. Clin Biochem 2011;44:771-8.

27. Horn PS, Pesce AJ, Copeland BE. A robust approach to reference interval estimation and evaluation. Clin Chem 1998;44:622-31.

28. Yang L, Grey V. Pediatric reference intervals for bone markers. Clin Biochem 2006;39:561-8.

29. Albertsson-Wikland K, Rosberg S, Karlberg J, Groth T. Analysis of 24-hour growth hormone profiles in healthy boys and girls of normal stature: relation to puberty. J Clin Endocrinol Metab 1994;78: 1195-201.

30. Klein KO, Martha PM Jr, Blizzard RM, Herbst T, Rogol AD. A longitudinal assessment of hormonal and physical alterations during normal puberty in boys. II. Estrogen levels as determined by an ultrasensitive bioassay. J Clin Endocrinol Metab 1996;81:3203-7.

31. Koivula MK, Risteli L, Risteli J. Measurement of aminoterminal propeptide of type I procollagen (PINP) in serum. Clin Biochem 2012;45:920-7.

32. Garnero P, Vergnaud P, Hoyle N. Evaluation of a fully automated serum assay for total $\mathrm{N}$-terminal propeptide of type I collagen in postmenopausal osteoporosis. Clin Chem 2008;54:188-96.

33. Eastell R, Garnero P, Audebert C, Cahall DL. Reference intervals of bone turnover markers in healthy premenopausal women: results from a cross-sectional European study. Bone 2012;50:1141-7.

34. Yoo JI, Park AJ, Lim YK, Kweon OJ, Choi JH, Do JH, et al. Age-related reference intervals for total collagen-I-N-terminal propeptide in healthy Korean population. J Bone Metab 2018;25:235-41. 